\title{
Antiretroviral therapy in HIV-infected patients: a proposal to assess the economic value of the single-tablet regimen
}

This article was published in the following Dove Press journal:

ClinicoEconomics and Outcomes Research

29 January 2013

Number of times this article has been viewed

\author{
Giorgio L Colombo ${ }^{1,2}$ \\ Sergio Di Matteo ${ }^{2}$ \\ Franco Maggiolo 3 \\ 'University of Pavia, Department \\ of Drug Sciences, School of Pharmacy, \\ Pavia, Italy, ${ }^{2}$ Studi Analisi Valutazioni \\ Economiche, Milan, Italy, ${ }^{3}$ Division \\ of Infectious Diseases, Ospedali \\ Riuniti, Bergamo, Italy
}

Background: The aim of this study was to assess the economic value of a reduced number of pills in patients infected with the immunodeficiency virus (HIV) and on highly active antiretroviral therapy by a cost-effectiveness model.

Methods: An incremental cost-effectiveness analysis of efavirenz, tenofovir, and emtricitabine (TDF-FTC-EFV) as a single-tablet regimen versus a multipill regimen, with reference to untreated HIV-infected patients, was carried out from the perspective of the Italian National Health Service. The comparisons were performed with the help of a Markov decision model over a 10-year time horizon. Based on the ADONE (ADherence to ONE pill) study, it was then possible to identify the utility score increment in patients switching from a multipill regimen of TDF-FTC + EFV therapy to a single-tablet regimen.

Results: The single-tablet regimen (0.755 quality-adjusted life-years [QALYs]/year) resulted in better patient quality of life, with a higher number of QALYs than for the TDF-FTC + EFV multipill regimen (0.716 QALYs/year). The single-tablet regimen was the most cost-effective treatment strategy, with an incremental cost-effectiveness ratio of $€ 22,017.00$ versus $€ 26,558.00$ for the multipill regimen. A $24 \%$ decrease in cost of the multipill regimen determined equivalence with the single-tablet regimen in terms of the incremental cost-effectiveness ratio. Univariate sensitivity and probabilistic analysis carried out on the main variables did not highlight significant variations with respect to the base case scenario.

Conclusion: The single-tablet regimen resulted in better adherence, and therefore better quality of life as perceived by patients, corresponding to a $€ 4541.00$ lower cost-effectiveness ratio per QALY versus the multipill regimen, with a $17 \%$ lower cost in favor of the singletablet regimen. The value determined could be used to identify a maximum potential "premium price" of $29 \%$ to be assigned to therapeutic regimens proposing a single-tablet regimen for HIV-infected patients.

Keywords: tenofovir, emtricitabine, efavirenz, single-tablet regimen, highly active antiretroviral therapy, quality of life, pharmacoeconomics, adherence

\section{Introduction}

Highly active antiretroviral therapy (HAART) has changed the clinical course of immunodeficiency virus (HIV) infection, decreasing the rate of disease progression, the incidence of opportunistic infections, and mortality. ${ }^{1}$ Owing to this prolonged survival, HIV infection can now be considered a chronic disease, ${ }^{2}$ with combination antiretroviral therapy resulting in longer survival and better quality of life for many HIV-infected patients. ${ }^{3}$ The therapeutic options currently available in Italy and Europe include over 20 approved antiretroviral drugs which are divided into five classes, ie, nucleoside/nucleotide reverse transcriptase inhibitors, non-nucleoside reverse
Correspondence: Giorgio L Colombo Studi Analisi Valutazioni Economiche, Via Previati, 74-20I49 Milan, Italy

Tel +3902485 I 9230

$\mathrm{Fax}+390273960369$

Email giorgio.colombo@unipv.it 
transcriptase inhibitors, protease inhibitors, fusion or entry inhibitors, and integrase inhibitors. Each of these groups attacks HIV via a different pathway. The most common HAART regimen administered to patients entering treatment consists of two nucleoside/nucleotide reverse transcriptase inhibitors combined with either a non-nucleoside reverse transcriptase inhibitor or a "boosted" protease inhibitor. ${ }^{4}$ Each drug varies greatly in terms of efficacy, resistance, pill burden, safety, adherence, and price. Researchers have recently contemplated the possibility of considering not only the simple prolongation of life expectancy, but also the quality of residual life for patients, with clear implications for clinical practice. In other words, they are wondering if it could be possible to increase survival without altering, or possibly further increasing, the quality of life. , $^{5,6}$

The recent development of the once-daily single-tablet regimen has been an important development in the optimization of antiretroviral regimens. ${ }^{4}$ Such optimization has the potential to improve long-term adherence, virologic efficacy, clinical outcomes, and quality of life. ${ }^{7}$ Optimization strategies using antiretroviral agents that are currently approved or under study include once-daily dosing regimens. Multiple studies have shown that adherence to antiretroviral therapeutic regimens is among the most powerful predictors of sustained virologic suppression, reduces the risk of developing drug resistance, limits disease progression, and improves patient survival..$^{8,9}$ In the past, several studies showed how treatment simplification strategies could enhance patient adherence with HAART. ${ }^{10-12}$ Although it has been postulated that this type of intervention works by improving patient quality of life, some studies have specifically addressed the relationship between quality of life and adherence. ${ }^{13}$ ADONE (ADherence to ONE pill) was a multicenter, open-label, comparative, prospective study with the objective of verifying if simplification of the antiretroviral regimen, measured as reduction in pill burden, might affect the patient adherence rate and quality of life. ${ }^{13}$ An important feature of this study is that the variables were evaluated without varying the drug components in the HAART regimens. All patients were on treatment with efavirenz in combination with tenofovir-emtricitabine or lamivudine-tenofovir which was simplified into a single-tablet regimen containing efavirenz, tenofovir, and emtricitabine (EFV-TDF-FTC), with the only modification being the number of pills needing to be taken daily. In this trial, the investigators evaluated how simplification of therapy could affect adherence, quality of life, and subjective perception of health status, and how these variables influenced one another. The patients did not change their therapy in terms of active ingredients or doses of the same molecules, but simply reduced the number of pills in their daily regimen from three or two to one. This simple change had a significant impact on quality of life and the patients' perception of their health in the subsequent 6 months. Therefore, the current objective of HAART is to prolong survival while guaranteeing and preserving quality of life, but there is a possible further development of its objectives in the future, based on the significant effectiveness of the HAART regimens and their tolerability, increased economic convenience, and consequent prolonged durability. For this reason, it seems important to verify how switching to a single-tablet regimen, which determines a simultaneous improvement in patient adherence and quality of life, may translate into a potential economic value as a result of the reduced number of pills needed in HIV-infected patients on HAART using a model of cost-effectiveness.

\section{Materials and methods}

We considered an economic analysis that estimates the cost-effectiveness of HAART regimens for managing HIV-infected patients versus untreated HIV-infected patients in Italy, based on Markov model simulation. ${ }^{14}$ The objective of the current study was to attribute a value to the difference in cost-effectiveness of a once-daily single-tablet regimen versus a multipill regimen, based on data on adherence and quality of life from the ADONE study. ${ }^{13}$

An incremental cost-effectiveness ratio (ICER) analysis was performed by applying a previously published Markov model ${ }^{14}$ enabling comparison of outcomes in HIV-infected patients treated with the study antiretroviral regimens versus untreated HIV-infected patients according to the Italian guidelines. ${ }^{4}$ In this analysis, the direct costs and effectiveness of each drug were compared with the direct costs and effectiveness of the natural history of the disease in the absence of treatment. ${ }^{15}$ The Markov model simulates quality of life and the cost of treating an HIV patient for ten years, starting from administration of the first dose, through one-year cycles, based on the antiretroviral therapy administered ${ }^{16}$ After entering the model and receiving one of the antiretroviral regimens, the patient can "move" through eight health states, defined by CD4 cell count and viremia levels, one acquired autoimmune deficiency syndrome (AIDS) state, and one death state (Figure 1). Modeling was undertaken using Microsoft Excel 2010 (Microsoft Corporation, Redmond, WA, USA).

The analysis was carried out from the point of view of the National Healthcare Service in Italy. The 


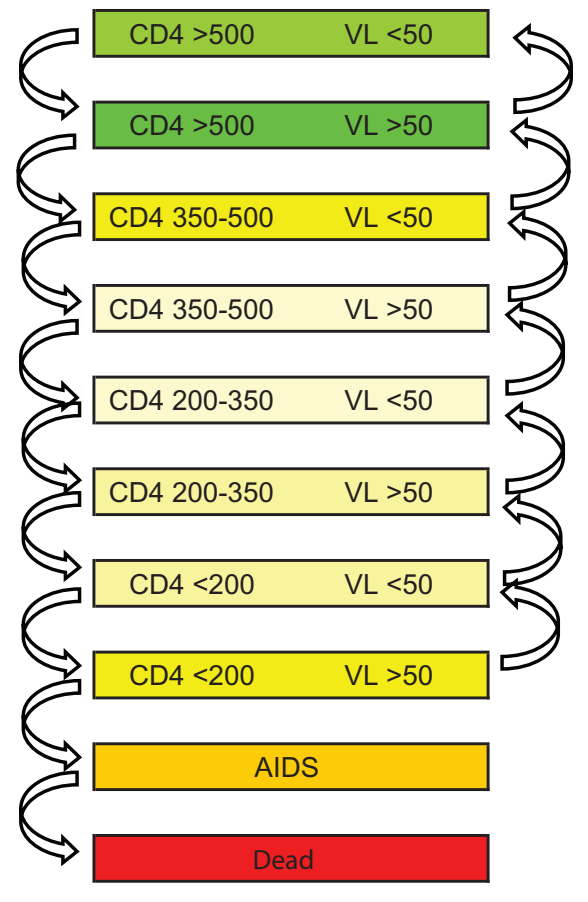

Figure I Structure of the Markov model.

Abbreviations: AIDS, acquired immunodeficiency syndrome; VL, viral load.

outcome measures considered were quality-adjusted life-years (QALYs), and the direct costs were updated for the year 2011. ${ }^{17}$ Both outcomes (QALYs) and costs were discounted by $3.5 \% .{ }^{18}$ The time horizon adopted in the model was ten years. The population considered in the analysis reflects typical patient characteristics, according to reports by one of the regional surveillance systems for HIV/AIDS infection currently operating in Italy. ${ }^{19}$

\section{Transition probabilities and quality of life}

The percentage distribution of patients considered in the model with respect to the proposed CD4 cell count takes into account observations on HIV infection/AIDS in Emilia Romagna (epidemics update as of December 31, 2009). The immunologic response to each of the two therapeutic study regimens, ie, TDF-FTC + EFV (multipill regimen) and the single-tablet regimen, was considered comparable, with $80 \%$ response rates in the first year, $67 \%$ in the second year, $64 \%$ in the third year, and $64 \%$ in the following years throughout ten observation years. ${ }^{20-22}$ When data were not available, it was assumed that the response remained constant at the last observed value by applying the last value carried forward technique.

QALYs were used as effectiveness indicators in this economic evaluation. The utility values associated with the eight health states identified by the CD4 cell count, as published in the report by Simpson et al, ${ }^{23}$ were calculated using the EQ-5D ${ }^{\circledR}$ questionnaire. ${ }^{24}$ The utility score was 0.9460 for CD4+ values $>500$ cells $/ \mu \mathrm{L} ; 0.9330$ for CD4+ $351-500$ cells $/ \mu \mathrm{L} ; 0.9310$ for $\mathrm{CD} 4+201-350$ cells $/ \mu \mathrm{L}$; and 0.8300 for $\mathrm{CD} 4+<200$ cells $/ \mu \mathrm{L}$.

Based on the study by Airoldi et al, ${ }^{13}$ it was possible to identify the utility score increment in patients switching from a twice-daily TDF-FTC + EFV multipill regimen to a oncedaily single-tablet TDF-FTC-EFV regimen. With regard to the reduction in number of pills needing to be taken daily from two to one, the ADONE study measured a 3.9\% increase in quality of life, from $68.8 \%$ (95\% confidence interval [CI] $67.7-70.0)$ to $72.7(95 \%$ CI $71.5-73.8, P=0.042)$ in health perceived after 6 months by patients who switched from a multipill regimen to a single-tablet regimen (Figure 2). The different utility values thus obtained for the response to the two therapeutic regimens were used to compare the costs of the two treatments versus those in untreated HIV-infected patients.

\section{Resource consumption and costs}

Resource consumption in the model was linked with administration of antiretroviral regimens (annual costs of $€ 7226.00)$ and other direct health care costs, including for hospitalizations, visits, and laboratory tests. The average annual costs for each first-line regimen and the purchase cost of the drugs were calculated based on the reimbursement price paid by the National Health Service, which takes into account price updates valid from January $1,2011 .{ }^{25}$ For every health state defined by the CD4 cell count, additional patient health care costs associated was assumed, including further consumption of health resources due to hospitalization, outpatient care, examinations by general practitioners and specialists, laboratory tests, and diagnostic procedures. These costs were estimated based on indications from studies published by Colombo et a ${ }^{14}$ and Garattini et al. ${ }^{17}$ The cost data stratified by CD4 count were then discounted to $2011 .{ }^{26}$

\section{Sensitivity analysis}

The sensitivity analysis modified some of the initial assumptions, namely the most uncertain or relevant ones, with the aim of verifying if the results obtained in the base case could be considered reliable enough to support rational decisions about resource allocation. ${ }^{27}$ Univariate, threshold value, and probabilistic sensitivity analyses were carried out. ${ }^{27,28}$ 


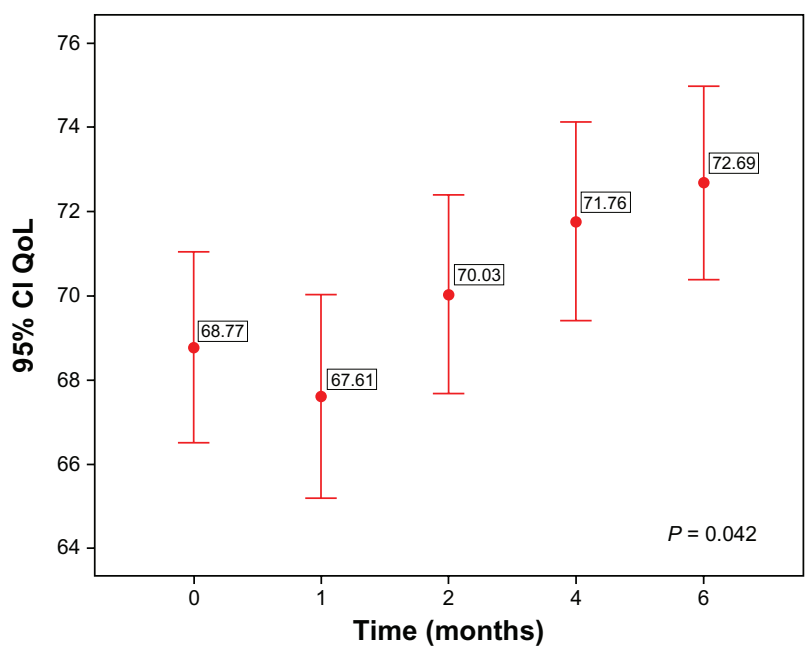

Figure 2 ADONE study. ${ }^{13}$

Note: Variation in quality of life self-reported by patients after switching from the TDF-FTC + EFV multipill regimen to the single-tablet regimen containing the same active ingredients.

Abbreviations: ADONE, ADherence to ONE pill study; $\mathrm{Cl}$, confidence interval; EFV, efavirenz; FTC, emtricitabine; QoL, quality of life; TDF, tenofovir.

The sensitivity analysis verified the impact of a series of variations in the base case scenario which had a considerable impact on the results obtained. ${ }^{15} \mathrm{~A}$ series of univariate analyses were carried out on some parameters of the simulation model, including variation in quality of life (utilities) and the cost of the single-tablet regimen, and identifying the threshold value for these parameters. In order to test the improvement in health state perceived by the patient from $68.8 \%$ to $72.7 \%$ (IC 95\%, $P=0.042$ ), a probabilistic sensitivity analysis was performed, using a normal distribution to evaluate the improvement in the patient's perceived health state. ${ }^{29}$ In order to obtain a variability measure of the study parameter, we obtained a bootstrap CI (percentile, bilateral, symmetrical) using the Monte Carlo method. One thousand casual values were extracted from the normal distribution. After determining the 1000 casual values of perceived health state, 1000 de novo utility values were calculated, and from these, the 1000 ICER was generated for the single-tablet regimen. Probabilistic sensitivity analysis was performed using TreeAge version 4.0 software (TreeAge Software Inc, Williamstown, MA).

\section{Results}

Table 1 shows the average annual cost and QALYs for a patient with HIV treated with each of the antiretroviral regimens, ie, the TDF-FTC + EFV multipill regimen versus the TDF-FTCEFV single-tablet regimen. The simulation model shows that the single-tablet regimen (0.755 QALYs/year) resulted in better patient adherence and quality of life, with a higher number of QALYs than with the daily TDF-FTC + EFV multipill regimen ( 0.716 QALYs/year). Table 1 shows that the average annual cost of the two treatments is equivalent $(€ 8551.00)$ and includes the therapeutic regimen cost (the purchase cost for the National Health Service is identical for the two regimens, ie, €7226.00) and other health care costs (hospitalizations, examinations, diagnostic tests). Based on the previously published model, ${ }^{14}$ it was finally possible to compare these two strategies with a no-treatment strategy in order to evaluate the contribution of the two therapeutic regimens in terms of ICER, ie, gained year of life weighted for quality (QALYs). After comparing the abovementioned costs and outcomes with the no-treatment strategy in incremental terms (ICER), the single-tablet regimen was shown to be the most cost-effective therapeutic choice, with an ICER of $€ 22,017.00$ versus $€ 26,558.00$, which is well below the threshold values considered to be acceptable. ${ }^{18}$

Therefore, this increment of about 0.039 points in QALY determines a $€ 4541.00$ (-17\%) improvement in the cost per QALY in favor of the single-tablet regimen. This amount corresponds to the potential economic value of a fixed combination, in terms of patient-reported improvement in quality of life versus the corresponding nonfixed combination regimen.

The sensitivity analysis carried out on the main variables does not highlight significant variations with respect to the base case scenario, as previously reported. ${ }^{14}$ However, it is interesting to note the threshold point analysis for the cost of the single-tablet regimen and changes in quality of life parameters. The threshold point analysis allows determination of the maximum values that invert the results for our base case. In Table 2, it is interesting to note that with a $1 \%$ increase in the single-tablet regimen cost from the current value, the two strategies are leveled at a single-tablet regimen price of $€ 9317.50$, corresponding to a $29 \%$ increase in the current market price. In other words, a $10 \%$ potential increase in the single-tablet regimen cost corresponds to a $7 \%$ increment in cost per QALY. Using a procedure similar to that used for the single-tablet regimen, it is possible to simulate a reduction in cost of the TDF-FTC + EFV multipill regimen to estimate the breakeven point in comparison with the single-tablet regimen. When the daily price of the multipill regimen is decreased by $24 \%$, the two formulations have the same ICER.

Table 3 shows an analysis of the threshold value for the potential increment of utilities (QALYs). For every $1 \%$ increase in utility value for the single-tablet regimen, there is a more than proportional increase in the ICER (3.51\%). 


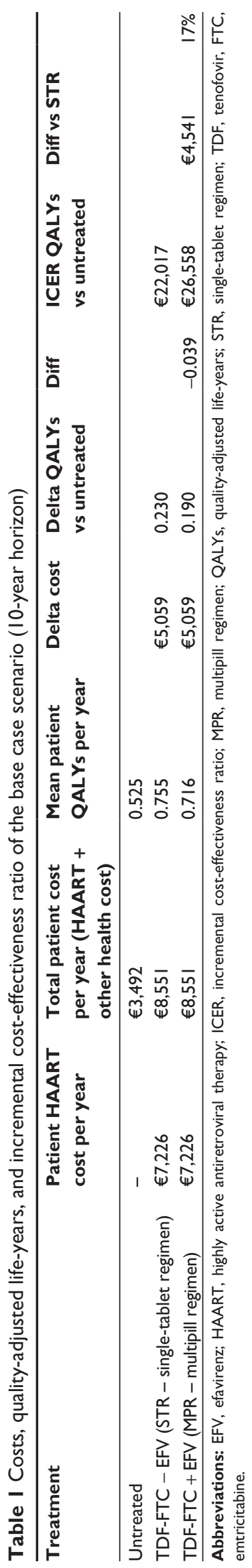

The results of the probabilistic sensitivity analysis were used to determine the cost function per QALY (ICER) by calculating the percentage of the 1000 iterations with the Monte Carlo simulation (shown on the $\mathrm{x}$ axis, or abscissa) which are lower than a certain cost per QALY (shown on the $y$ axis, or ordinate). Such a percentage corresponds to the estimated probability that the cost per QALY for the single-tablet regimen is lower than the per QALY cost for the multipill regimen (Figure 2). Figure 3 shows that, for all 1000 simulation values, the ICER generated by the single-tablet regimen is consistently lower in probabilistic terms than the one generated by the daily multipill regimen.

\section{Discussion}

Adherence to chronic therapies is a widespread problem in all medical disciplines. In 2003, the World Health Organization reported that $30 \%-50 \%$ of medications prescribed for chronic diseases are not taken according to the instructions given to patients. ${ }^{30}$ Multiple studies have demonstrated that adherence with antiretroviral therapeutic regimens is one of the most powerful predictors of sustained virologic suppression, reduces the risk of drug resistance, limits disease progression, and improves patient survival and quality of life. ${ }^{13,31-33}$ Analysis of a Swiss cohort ${ }^{34}$ highlighted that $30 \%$ of patients reported omitting one or more drug doses in the preceding weeks and that $7.1 \%$ of the same patients reported less than $95 \%$ adherence. Among the factors that negatively influenced the levels of adherence, use of a protease inhibitor with a booster was statistically significant. Another study ${ }^{35}$ found higher adherence in patients treated with non-nucleoside reverse transcriptase inhibitors (93.6\%) compared with those who received a regimen based on three nucleoside reverse transcriptase inhibitors $(91.7 \%)$ or protease inhibitors $(89.9 \%)$ with or without booster. Finally, in a study using a microelectromechanical monitoring system, ${ }^{36}$ the investigators observed an adherence rate of $91.5 \%$ and $86 \%$ in respect of administration timetables for protease inhibitors only. Substantial literature is available in which various authors have analyzed the relationship between adherence and number of pills needing to be taken. ${ }^{10,35-39}$ An evaluation of the impact of this variable on adherence was reported by Maggiolo et al, ${ }^{40}$ showing that "too many pills" was reported by $20 \%$ of patients to justify their reduced adherence and how the daily number of pills needed correlated significantly $(P=0.021)$ with adherence. In particular, the simplest therapies, ie, those 
Table 2 Results of one-way sensitivity analyses: variation in annual cost of single-tablet regimen and incremental cost-effectiveness ratio (ICER)

\begin{tabular}{|c|c|c|c|c|c|c|c|}
\hline $\begin{array}{l}\text { STR cost } \\
\text { per year }\end{array}$ & $\%$ increase & ICER & $\%$ increase & $\begin{array}{l}\text { TDF-FTC + EFV (MPR) } \\
\text { cost per year }\end{array}$ & $\%$ decrease & ICER & $\%$ decrease \\
\hline$€ 7,226$ & $0 \%$ & 22,017 & $0 \%$ & 7,226 & $0 \%$ & 26,558 & $0 \%$ \\
\hline$€ 7,298$ & $1 \%$ & 22,173 & $1 \%$ & 7,154 & $-1 \%$ & 26,366 & $-1 \%$ \\
\hline$€ 7,371$ & $2 \%$ & 22,330 & $1 \%$ & 7,081 & $-2 \%$ & 26,178 & $-1 \%$ \\
\hline$€ 7,443$ & $3 \%$ & 22,486 & $2 \%$ & 7,009 & $-3 \%$ & 25,990 & $-2 \%$ \\
\hline$€ 7,5 । 5$ & $4 \%$ & 22,642 & $3 \%$ & 6,937 & $-4 \%$ & 25,801 & $-3 \%$ \\
\hline$€ 7,587$ & $5 \%$ & 22,799 & $4 \%$ & 6,865 & $-5 \%$ & 25,612 & $-4 \%$ \\
\hline$€ 7,660$ & $6 \%$ & 22,955 & $4 \%$ & 6,792 & $-6 \%$ & 25,423 & $-4 \%$ \\
\hline$€ 7,732$ & $7 \%$ & 23,111 & $5 \%$ & 6,720 & $-7 \%$ & 25,234 & $-5 \%$ \\
\hline$€ 7,804$ & $8 \%$ & 23,267 & $6 \%$ & 6,648 & $-8 \%$ & 25,045 & $-6 \%$ \\
\hline$€ 7,876$ & $9 \%$ & 23,424 & $6 \%$ & 6,576 & $-9 \%$ & 24,856 & $-6 \%$ \\
\hline$€ 7,949$ & $10 \%$ & 23,580 & $7 \%$ & 6,503 & $-10 \%$ & 24,667 & $-7 \%$ \\
\hline$€ 8,021$ & $11 \%$ & 23,736 & $8 \%$ & 6,431 & $-11 \%$ & 24,478 & $-8 \%$ \\
\hline$€ 8,093$ & $12 \%$ & 23,893 & $9 \%$ & 6,359 & $-12 \%$ & 24,289 & $-9 \%$ \\
\hline$€ 8,165$ & $13 \%$ & 24,049 & $9 \%$ & 6,287 & $-13 \%$ & 24,100 & $-9 \%$ \\
\hline$€ 8,238$ & $14 \%$ & 24,205 & $10 \%$ & 6,214 & $-14 \%$ & 23,912 & $-10 \%$ \\
\hline$€ 8,310$ & $15 \%$ & 24,362 & $11 \%$ & 6,142 & $-15 \%$ & 23,723 & $-11 \%$ \\
\hline$€ 8,382$ & $16 \%$ & 24,518 & $11 \%$ & 6,070 & $-16 \%$ & 23,534 & $-11 \%$ \\
\hline$€ 8,454$ & $17 \%$ & 24,674 & $12 \%$ & 5,998 & $-17 \%$ & 23,345 & $-12 \%$ \\
\hline$€ 8,527$ & $18 \%$ & 24,830 & $13 \%$ & 5,925 & $-18 \%$ & 23,156 & $-13 \%$ \\
\hline$€ 8,599$ & $19 \%$ & 24,987 & $13 \%$ & 5,853 & $-19 \%$ & 22,967 & $-14 \%$ \\
\hline$€ 8,67 \mid$ & $20 \%$ & 25,143 & $14 \%$ & $5,78 \mid$ & $-20 \%$ & 22,778 & $-14 \%$ \\
\hline$€ 8,743$ & $21 \%$ & 25,299 & $15 \%$ & 5,709 & $-21 \%$ & 22,589 & $-15 \%$ \\
\hline$€ 8,816$ & $22 \%$ & 25,456 & $16 \%$ & 5,636 & $-22 \%$ & 22,400 & $-16 \%$ \\
\hline$€ 8,888$ & $23 \%$ & 25,612 & $16 \%$ & 5,564 & $-23 \%$ & 22,211 & $-16 \%$ \\
\hline$€ 8,960$ & $24 \%$ & 25,768 & $17 \%$ & 5,492 & $-24 \%$ & 22,017 & $-17 \%$ \\
\hline$€ 9,033$ & $25 \%$ & 25,925 & $18 \%$ & 5,420 & $-25 \%$ & 21,834 & $-18 \%$ \\
\hline$€ 9,105$ & $26 \%$ & 26,081 & $18 \%$ & 5,347 & $-26 \%$ & 21,645 & $-19 \%$ \\
\hline$€ 9,177$ & $27 \%$ & 26,237 & $19 \%$ & 5,275 & $-27 \%$ & 21,456 & $-19 \%$ \\
\hline$€ 9,249$ & $28 \%$ & 26,393 & $20 \%$ & 5,203 & $-28 \%$ & 21,267 & $-20 \%$ \\
\hline$€ 9,322$ & $29 \%$ & 26,550 & $21 \%$ & 5,130 & $-29 \%$ & 21,078 & $-21 \%$ \\
\hline$€ 9,318$ & $29 \%$ & 26,558 & $21 \%$ & 5,058 & $-30 \%$ & 20,889 & $-21 \%$ \\
\hline$€ 9,394$ & $30 \%$ & 26,706 & $21 \%$ & 4,986 & $-31 \%$ & 20,700 & $-22 \%$ \\
\hline
\end{tabular}

Abbreviations: EFV, efavirenz; ICER, incremental cost-effectiveness ratio; MPR, multipill regimen; STR, single-tablet regimen; TDF, tenofovir; FTC, emtricitabine.

that need to be administered only once a day, invariably correlated with significantly $(P=0.009)$ higher adherence levels. ${ }^{35}$ Finally, several authors have reported a close link between adherence rate and risk of virologic failure. ${ }^{41-44}$ Recent studies have shown that HAART regimens based on only one tablet a day (ie, a single-tablet regimen) are associated with significantly better adherence and a lower hospitalization risk in HIV patients in comparison with patients treated with more complicated regimens involving three or more tablets a day. ${ }^{45}$ In this context, the once-daily TDF-FTC-EFV single-tablet regimen appears to be a reasonable option for individuals with multiple barriers to adherence ${ }^{46}$ and, although new antiretroviral drugs and classes have become available in recent years, the single-tablet regimen continues to show an advantage in improving adherence in comparison with other combination antiretroviral therapies. ${ }^{47}$

Given that simpler antiretroviral regimens are considered easier to follow and result in improved patient adherence, the trend over the last decade has been to simplify treatment regimens as far as possible. ${ }^{4}$ From this point of view, a multicenter study was carried out in Italy ${ }^{13}$ exploiting the availability of a fixed-dose single-tablet regimen. In this simplification study, patients treated with the individual components of the single-tablet regimen switched to taking the single-tablet regimen itself, thus changing only the number of pills but without modifying the pharmacologic content of the therapeutic regimen. While the patients took the single components, adherence (as a percentage of doses taken) was 96.1\% (95\% CI 90.4-95.4) but increased to 97.1\% (CI 97.0- 


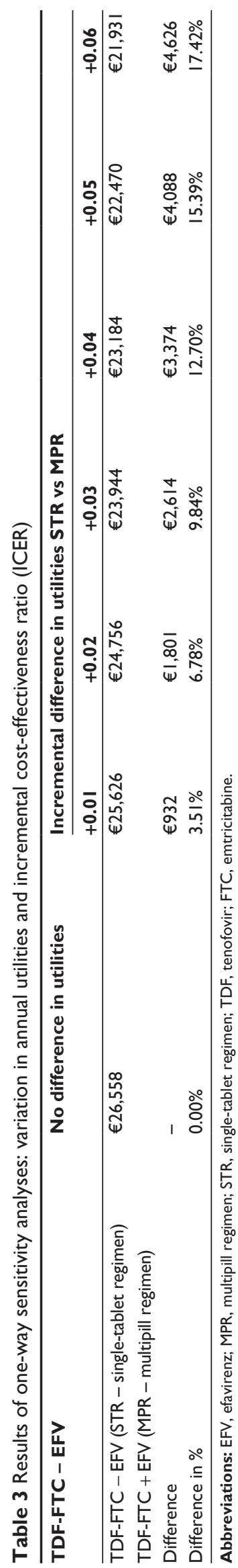

98.4, $P=0.014)$ after the patients switched to the single-tablet regimen. Adding new single-tablet antiretroviral regimens to conventional therapies can help physicians in the choice of the best possible treatment to administer to HIV patients. Because the single-tablet regimen is not the only available therapeutic alternative, it was deemed necessary to carry out a comparison with other antiretroviral regimens containing the same active ingredients, but not in fixed combination, with the aim of highlighting the presence of potential benefits for patients, and ultimately, for the Italian National Health Service. Specifically, an incremental cost-effectiveness analysis of TDF-FTC-EFV in the single-tablet regimen versus the TDF-FTC + EFV multipill regimen was performed in comparison with untreated HIV-infected patients from the point of view of the National Health Service. ${ }^{14}$ Using the Markov model, we could identify the economic value of the improved response by patients on the single-tablet regimen versus those on a multipill regimen in terms of quality of life and cost per QALY gained. As a result of the better quality of life perceived by patients, the ICER was more favorable and came to $€ 4541.00$ per QALY (-17\%). In practice, assessment of the single-tablet regimen in the terms described here may indicate the range of cost reduction possible for a treatment regimen comprising various molecules (some of which may be nearing patent expiration) with a cost-effectiveness ratio equivalent to that of the corresponding single-tablet regimen. In our study, a $24 \%$ price decrease for the multipill regimen would make it comparable with that of the single-tablet regimen. Similarly, the results of our study could be used to identify a potential premium price to be assigned to a singletablet regimen. In the proposed study, the maximum premium price could be about $29 \%$ of the corresponding noncombined therapeutic regimen.

Our study has a few limitations, the most important being the quality of data entered into the model, with parameters such as efficacy and utilities scores, for example, being based on studies of relatively short duration, which may be inadequate for modeling the treatment of a chronic disease for a longer period of time. In our analysis, we include results from a visual analog scale (Short Form-36 $6^{13}$ ) in order to weight the utility scores for patients treated with the single-tablet regimen. In this case, the best solution would be to weight utilities on the basis of the data measured, eg, with the EQ5D; however, in the absence of these data, we weighted the utilities taking account of some positive relationships between these two assessment tools when used in other diseases. ${ }^{48,49}$ For this reason, changes in the utilities deriving from the switch to a single-tablet regimen were examined using a 


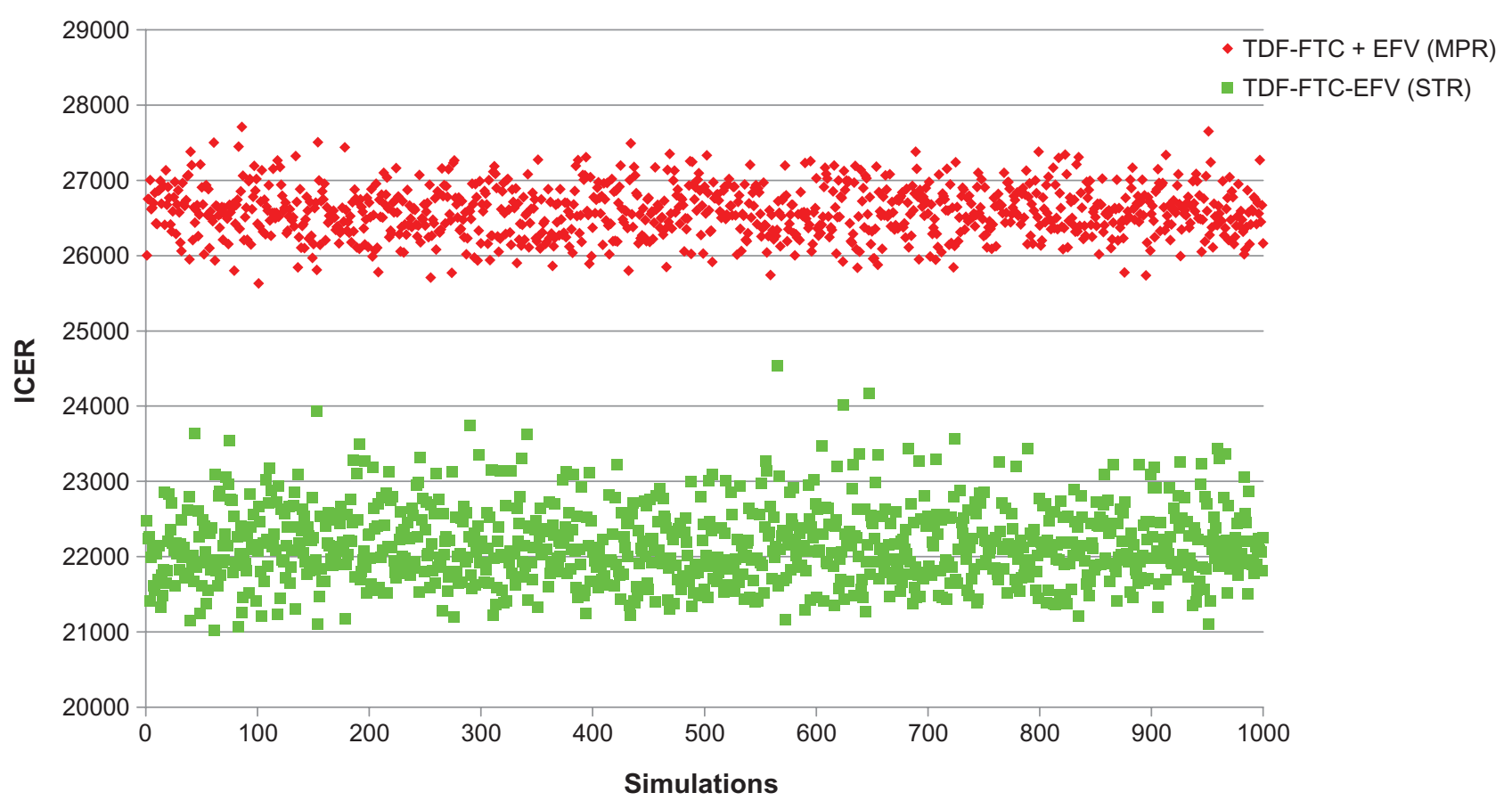

Figure 3 Probabilistic sensitivity analysis: variation in incremental cost-effectiveness ratio for TDF-FTC + EFV multipill regimen versus single-tablet regimen. Abbreviations: EFV, efavirenz; FTC, emtricitabine; STR, single-tablet regimen; TDF, tenofovir; ICER, incremental cost-effectiveness ratio.

rigorous probabilistic sensitivity analysis which took into account their limitations and ranges of variation.

Another important limitation is the assumptions on which the analysis is based, that may be necessary to simplify the model or in case of incomplete data. Specifically, transition probabilities were lacking in some cases, and were thus assumed to remain constant over time, and the utilities were derived from different literature sources and considered to be acceptable for an Italian population. ${ }^{14}$ Therefore, such models can be used to predict the possible final results, such as in our case, when the clinical trial is incomplete or lacking important data (in this case, the data necessary for the economic evaluation was derived from different and nonhomogeneous sources) and only measures an intermediate result or has a short-period follow-up. For example, the improvement in quality of life seen upon switching from a multipill regimen to a single-tablet regimen was only assessed over a one-year time horizon, whereas the results of our study were extended to 10 years of assessment, for reasons of economic simulation. This hypothesis is based on the assumption that the results of the study by Airoldi et al ${ }^{13}$ were also obtained over longer time periods. However, a recent paper $^{48}$ observes that the once-daily single-tablet regimen showed greater durability than the twice-daily multipill regimen during an observation period of 7 years. For the single-tablet regimen and multipill regimen, the durability, time from initiation of antiretroviral therapy to first treatment modification, after a reduction of first 48 months, remains constant in this period of observation. Once-daily single-tablet regimens have shown greater durability than twice-daily multipill regimens. ${ }^{50}$

Acknowledging the abovementioned limitations, the results of this study allow us to propose a pharmacoeconomic pathway for assigning a value to combination HIV therapies aimed at simplifying daily treatment into a single-tablet regimen. This pathway runs through the development of a series of epidemiologic and cost-of-illness studies, as well as simulation models, which are necessary when obtaining a picture of patients, costs, their evolution over time, and clinical studies to assess and compare the various treatment options, including quality of life. The information contained in this type of study should be summarized in pharmacoeconomic simulation models to provide constant support to policy-makers, because decisions about resource allocation (price and drug reimbursement) must be made before introduction of new treatments on the market and, therefore, are based on hypotheses and simulations that can only be validated by the presence of the drug on the market. In spite of the limitations of pharmacoeconomic models, these tools play a fundamental role when it is necessary to establish priorities in allocation of resources. Indeed, they supply decision-makers within health care systems with useful tools to make more rational and effective decisions. By collecting appropriate data and integrating them into the 
abovementioned models, it is possible to obtain more accurate estimates for the cost-effectiveness ratios of the various strategies used in the treatment of immunocompromised patients and to supply significant evidence for use in future prospective pharmacoeconomic evaluations.

\section{Disclosure}

This research was supported by Gilead Sciences Srl, Milan, Italy. The authors are employees of independent research organizations and maintained independent scientific control over the study, including data analysis and interpretation of the final results.

\section{References}

1. The Antiretroviral Therapy Cohort Collaboration. Life expectancy of individuals on combination antiretroviral therapy in high-income countries: a collaborative analysis of 14 cohort studies. Lancet. 2008;372: 293-299.

2. Volberding PA, Deeks SG. Antiretroviral therapy and management of HIV infection. Lancet. 2010;376:49-62.

3. Freedberg KA, Losina E, Weinstein MC, et al. The cost effectiveness of combination antiretroviral therapy for HIV disease. $N$ Engl J Med. 2001;344:824-831.

4. Ministry of Health, National Committee on AIDS. Linee Guida Italiane sull'utilizzo dei farmaci antiretrovirali e sulla gestione diagnosticoclinica delle persone con infezione da HIV-1 -Luglio 2012. Italian.

5. Burgoyne RW, Tan DH. Prolongation and quality of life for HIV-infected adults treated with highly active antiretroviral therapy (HAART): a balancing act. J Antimicrob Chemother. 2008;61:469-473.

6. Camacho R, Teófilo E. Antiretroviral therapy in treatment-naïve patients with HIV infection. Curr Opin HIV AIDS. 2011;6 Suppl 1:S3-S11.

7. Nachega JB, Rosenkranz B, Pham PA. Twice-daily versus oncedaily antiretroviral therapy and coformulation strategies in HIVinfected adults: benefits, risks, or burden? Patient Prefer Adherence. 2011;5:645-651.

8. Parienti JJ, Bangsberg DR, Verdon R, Gardner EM. Better adherence with once-daily antiretroviral regimens: a meta-analysis. Clin Infect Dis. 2009;48:484-488.

9. Pulido F, Ribera E, Moreno S, et al. Once-daily antiretroviral therapy: Spanish consensus statement. J Antimicrob Chemother. 2005;56: 808-818.

10. Maggiolo F, Ripamonti D, Arici C, et al. Simpler regimens may enhance adherence to antiretrovirals in HIV infected patients. HIV Clin Trials. 2002;5:371-378.

11. Stone VE, Jordan J, Tolson J, et al. Perspectives on adherence and simplicity of HIV-infected patients on antiretroviral therapy. Self-report of the relative importance of multiple attributes of highly active antiretroviral therapy (HAART) regimens in predicting adherence. J Acquir Immune Defic Syndr. 2004;36:808-816.

12. Boyle B, Jayaweera D, Witt MD, et al. Randomization to once-daily stavudine extended release/lamivudine/efavirenz versus more frequent regimen improves adherence while maintaining viral suppression. HIV Clin Trials. 2008;9:164-176.

13. Airoldi M, Zaccarelli M, Bisi L, et al. One-pill once-a-day HAART: a simplification strategy that improves adherence and quality of life of HIV-infected subjects. Patient Prefer Adherence. 2010;4:115-125.

14. Colombo GL, Colangeli V, Di Biagio A, Di Matteo S, Viscoli C, Viale P. Cost-effectiveness analysis of initial HIV treatment under Italian guidelines. Clinicoecon Outcomes Res. 2011;3:197-205.

15. Drummond MF, O'Brien BJ, Stoddart GL, et al. Methods for the Economic Evaluation of Health Care Programmes, 3rd ed. New York, NY: Oxford University Press; 2006.
16. Simpson KN, Strassburger A, Jones WJ, Dietz B, Rajagopalan R. Comparison of Markov model and discrete-event simulation techniques for HIV. Pharmacoeconomics. 2009;27:159-165.

17. Garattini L, Tediosi F, Di Cintio E, Yin D, Parazzini F; Gruppo di Studio ARCA (AIDS Resources and Costs Analysis). Resource utilization and hospital cost of HIV/AIDS care in Italy in the era of highly active antiretroviral therapy. AIDS Care. 2001;13:733-741.

18. Italian Health Economics Association. Italian guidelines proposal on how to conduct economic evaluation studies of health programs. Pharmacoeconomics. 2009;11:83-93. Italian.

19. Regione Emilia-Romagna. Lo stato dell'infezione da hiv/aids in emilia-romagna Aggiornamento sull'epidemia e primi risultati del sistema di sorveglianza dell'infezione da HIV al 31/12/2008. Italian.

20. Gallant JE, DeJesus E, Arribas JR, et al; Study 934 Group. Tenofovir DF, emtricitabine, and efavirenz vs zidovudine, lamivudine, and efavirenz for HIV. N Engl J Med. 2006;354:251-260.

21. Pozniak AL, Gallant JE, DeJesus E, et al. Tenofovir disoproxil fumarate, emtricitabine, and efavirenz versus fixed-dose zidovudine/lamivudine and efavirenz in antiretroviral-naive patients virologic, immunologic, and morphologic changes - a 96-week analysis. J Acquir Immune Defic Syndr. 2006;43:535-540.

22. Arribas JR, Pozniak AL, Gallant JE, et al. Tenofovir disoproxil fumarate, emtricitabine, and efavirenz compared with zidovudine/lamivudine and efavirenz in treatment-naive patients: 144-week analysis. J Acquir Immune Defic Syndr. 2008;47:74-78.

23. Simpson KN, Luo MP, Chumney E, et al. Cost-effectiveness of lopinavir/ritonavir versus nelfinavir as the first-line highly active antiretroviral therapy regimen for HIV irtfection. HIV Clin Trials. 2004;5:294-304.

24. http://www.euroqol.org. Accessed May 2012.

25. AIFA Italian Medicines Agency. Available from: http://www.agenziafarmaco. gov.it/it/content/negoziazione-e-rimborsabilit\%C3\%A0. Accessed May 2012.

26. ISTAT. Availbale from: http://www.istat.it/prezzi/precon/dati/. Accessed May 2012.

27. Gold MR, Siegel JE, Russel LB, Weinstein MC, editors. Costeffectiveness in Health and Medicine. New York, NY: Oxford University Press; 1996.

28. Briggs AH, Gray AM. Handling uncertainty when performing economic evaluation of health care interventions. Health Technol Assess. 1999;3: $1-134$.

29. Briggs AH. Handling uncertainty in economic evaluation and presenting the results. In: Drummond M, McGuire A, editors. Economic Evaluation in Health Care. Merging Theory with Practice. Oxford, UK: Oxford University Press; 2001.

30. World Health Organization. Adherence to long-term therapies: evidence for action. WHO report MNC/03/01. Available from: http://www. who.int/chp/knowledge/publications/adherence_report/en/. Accessed December 7, 2012.

31. Bangsberg DR, Hecht FM, Charlebois ED, et al. Adherence to protease inhibitors, HIV-1 viral load, and development of drug resistance in an indigent population. AIDS. 2000;14:357-366.

32. Bangsberg DR, Perry S, Charlebois ED, et al. Non-adherence to highly active antiretroviral therapy predicts progression to AIDS. AIDS. 2001;15:1181-1183.

33. Bartlett JA, Fath MJ, Demasi R, et al. An updated systematic overview of triple combination therapy in antiretroviral-naive HIV-infected adults. AIDS. 2006;20:2051-2064.

34. Glass TR, De Geest S, Weber R, et al. Correlates of self-reported non-adherence to antiretroviral therapy in HIV-infected patients. The Swiss HIV Cohort study. J Acquir Immune Defic Syndr. 2006;41: 385-392.

35. Maggiolo F, Ravasio L, Ripamonti D, et al. Similar adherence rates favour different virologic outcomes for patients treated with nonnucleoside analogues or protease inhibitors. Clin Infect Dis. 2005;40:158-163. 
36. Deschamps AE, De Graeve V, Van Wijngaerden E, et al. Prevalence and correlates of nonadherence to antiretroviral therapy in a population of HIV patients using medication event monitoring system. AIDS Patient Care STDS. 2004;18:644-657.

37. Diabate S, Alary M, Koffi CK. Determinants of adherence to highly active antiretroviral therapy among HIV-1-infected patients in Cote d'Ivoire. AIDS. 2007;21:1799-1803.

38. Stone VE, Jordan J, Tolson J, Miller R, Pilon T. Perspectives on adherence and simplicity of HIV-infected patients on antiretroviral therapy. Self-report of the relative importance of multiple attributes of highly active antiretroviral therapy (HAART) regimens in predicting adherence. J Acquir Immune Defic Syndr. 2004;36:808-816.

39. Lima VD, Harrigan R, Bangsberg DR, et al. The combined effect of modern highly active antiretroviral therapy regimens and adherence on mortality over time. J Acquir Immune Defic Syndr. 2009;50:529-536.

40. Maggiolo F, Ripamonti D, Arici C, et al. Simpler regimens may enhance adherence to antiretrovirals in HIV infected patients. HIV Clin Trials. 2002;5:371-378.

41. Parienti JJ, Massari V, Descamps D, et al. Predictors of virologic failure and resistance in HIV-infected patients treated with nevirapine- or efavirenz-based antiretroviral therapy. Clin Infect Dis. 2004;38:1311-1316.

42. Paterson DL, Swindells S, Mohr J, et al. Adherence to protease inhibitor therapy and outcomes in patients with HIV infection. Ann Intern Med. 2000;133:21-30.

43. Trotta MP, Ammassari A, Cozzi-Lepri A, et al. Adherence to highly active antiretroviral therapy is better in patients receiving non-nucleoside reverse transcriptase inhibitor-containing regimens than those receiving protease inhibitor-containing regimens. AIDS. 2003;17:1099-1102.
44. Le Moing V, Chene G, Carrieri MP, et al. Predictors of virological rebound in HIV-1-infected patients initiating a protease inhibitorcontaining regimen. AIDS. 2002;16:21-29.

45. Sax PE, Meyers JL, Mugavero M, et al. Adherence to antiretroviral treatment and correlation with risk of hospitalization among commercially insured HIV patients in the United States. PLoS One. 2012; 7:e31591.

46. Bangsberg DR, Ragland K, Monk A, Deeks SG. A single tablet regimen is associated with higher adherence and viral suppression than multiple tablet regimens in HIV+ homeless and marginally housed people. AIDS. 2010;24:2835-2840.

47. Sterrantino G, Santoro L, Bartolozzi D, et al. Self-reported adherence supports patient preference for the single tablet regimen (STR) in the current cART era. Patient Prefer Adherence. 2012;6:427-433.

48. Woo G, Tomlinson G, Yim C, et al. Health state utilities and quality of life in patients with hepatitis B. Can J Gastroenterol. 2012;26: $445-451$.

49. Buitinga L, Braakman-Jansen LM, Taal E, et al. Comparative responsiveness of the EuroQol-5D and Short Form 6D to improvement in patients with rheumatoid arthritis treated with tumor necrosis factor blockers: results of the Dutch Rheumatoid Arthritis Monitoring registry. Arthritis Care Res (Hoboken). 2012;64:826-332.

50. Di Biagio A, Prinapori R, Giannarelli D, et al; on behalf of the ARCA Collaborative Group. Duration of first-line antiretroviral therapy with tenofovir and emtricitabine combined with atazanavir/ritonavir, efavirenz or lopinavir/ritonavir in the Italian ARCA cohort. JAntimicrob Chemother. August 25, 2012. [Epub ahead of print.]
ClinicoEconomics and Outcomes Research

\section{Publish your work in this journal}

ClinicoEconomics \& Outcomes Research is an international, peerreviewed open-access journal focusing on Health Technology Assessment, Pharmacoeconomics and Outcomes Research in the areas of diagnosis, medical devices, and clinical, surgical and pharmacological intervention. The economic impact of health policy and health systems

\section{Dovepress}

organization also constitute important areas of coverage. The manuscript management system is completely online and includes a very quick and fair peer-review system, which is all easy to use. Visit http://www.dovepress.com/testimonials.php to read real quotes from published authors. 